\title{
RECENT DEVELOPMENTS OF INSERTION DEVICES AT THE ESRF
}

\author{
J. Chavanne, P. Elleaume, P. Van Vaerenbergh \\ ESRF, B.P.220, F-38043 GRENOBLE Cedex France
}

\begin{abstract}
57 Insertion Devices (IDs) segments are now in operation in the ESRF Storage Ring serving 29 beamlines. A review of the present situation is made together with a detailed description of the recently built in-vacuum undulator. The mechanical and magnetic designs together with the pumping and conditioning with beam are described. No lifetime reduction is observed for any magnetic gap larger than $6 \mathrm{~mm}$. A summary is made of the most important properties of several new innovative IDs including the $3 \mathrm{~T}$ permanent magnet wiggler, the quasi-periodic undulator and the fast switching helical undulator.
\end{abstract}

\section{INTRODUCTION}

The European Synchrotron Radiation Facility (ESRF) is a third generation synchrotron light source optimized to produce high brilliance X-rays in the $1-100 \mathrm{keV}$ range by means of Insertion Devices. The IDs consist of $1.6 \mathrm{~m}$ long segments. A complete $5 \mathrm{~m}$ long straight section accommodates three segments. At present, 57 segments are in operation representing a cumulative length of $90 \mathrm{~m}$ and serving 29 ID beamlines. The IDs are built according to the specific requirements of each beamline user. As a result, more than 30 different magnetic designs have been implemented. Almost all IDs are made of permanent magnets with magnet blocks in the open air on both sides of a fixed aperture vacuum chamber. More than $80 \%$ of IDs are low field undulators which provide a record brilliance of up to $1.5 \times 10^{20} \mathrm{Phot} / \mathrm{s} / 0.1 \% / \mathrm{mm}^{2} / \mathrm{mrad}^{2}$. Due to a mechanical and magnetic shimming process, the rms phase errors of the large majority of conventional undulators are in the 1-2 degree range at any useful gap, allowing safe operation on high harmonic numbers. The high energy range above $40 \mathrm{keV}$ is covered by wigglers with a moderate brilliance. In an attempt to shift the undulator spectrum to higher energies, an in-vacuum undulator has been built and is now in operation. It is described in detail in Section 2. Other new special IDs are briefly discussed in Section 3, such as the 3 Tesla permanent magnet wiggler, the quasi-periodic undulator and the fast switching helical undulator.

\section{IN-VACUUM UNDULATOR}

An in-vacuum undulator, designed and built at the ESRF, was installed in the storage ring during the 1999 January shutdown. The commissioning took only a few days and less than a week after it had received the first beam, it was in use on the Material Science beamline, ID11.

\subsection{Mechanical design of the carriage}

The carriage and its vacuum system is shown in Fig.1.

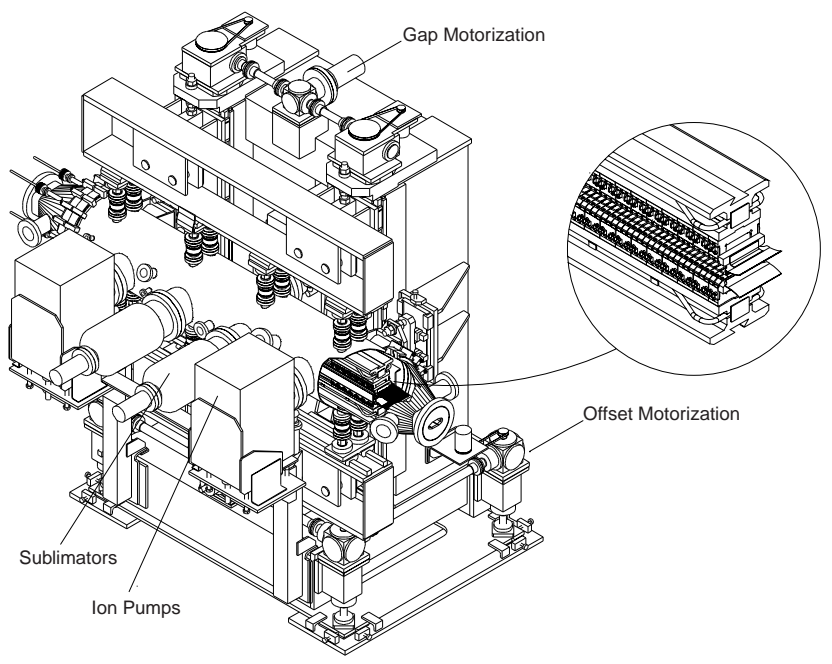

Figure 1: In-vacuum undulator carriage and vacuum system

This presents two remotely-controlled motorizations. The main one is dedicated to the magnetic gap tuning ranging from 0 to $30 \mathrm{~mm}$ at a maximum speed of $1 \mathrm{~mm} / \mathrm{s}$ and a resolution of $0.6 \mu \mathrm{m}$. The other (the offset) vertically translates the full magnet assembly for a precise centering of the electron beam in the middle of the gap of the undulator in order to maximize the beam lifetime at small gaps. The offset, rarely used, enables an immediate re-alignment following any possible ground settlement. Stepper motors and absolute encoders are used to drive and monitor each motion. To minimize the size of the vacuum tank, a rigid girder is placed in the open air on the outside of the vacuum. This minimizes the deformation under the magnetic load. A smaller cross-section girder is located in the vacuum. Both girders are linked to each other by eight connecting rods equipped with UHV bellows.

\subsection{Magnets}

The magnet structure is a hybrid structure with 69 periods of $23 \mathrm{~mm}$. It is expected that in extreme situations the gap will be the limiting aperture. This may result in a possible scraping of the electron beam by the magnetic assemblies. To minimize any risk of demagnetization from exposure to the electron beam [1] a grade of $\mathrm{Sm}_{2} \mathrm{Co}_{17}$ (INCOR26, produced by UGIMAG) was used for the magnet instead of the $\mathrm{NdFeB}$ material used in conventional undulators. The magnetic properties of 
$\mathrm{Sm}_{2} \mathrm{Co}_{17}$ are more resistant to high temperature and to irradiation by electrons. The penalty is a $15 \%$ reduction in magnetization. The magnet blocks are protected by a Nickel galvanic coating (10 $\mu \mathrm{m}$ thick) which simplifies the cleaning of the magnet surfaces. Following the delivery of the magnet blocks and soft iron poles (ARMCO steel), a special cleaning sequence [2] was applied: degreasing with Perchloroethylene vapour (at 120 $\left.{ }^{\circ} \mathrm{C}\right)$, followed by an ultrasonic cleaning in an alkaline bath (at $60{ }^{\circ} \mathrm{C}$ ), and finally rinsing with deionised water in an ultrasonic bath (at $60{ }^{\circ} \mathrm{C}$ ). As a result of this cleaning, a small magnetization loss of $0.25 \%$ was measured. Following the assembly of magnet and poles, and after magnetic correction, a $\mathrm{Cu}-\mathrm{Ni}$ sheet (i.e. a $50 \mu \mathrm{m}$ thick ribbon of $\mathrm{Ni}$, plated with a deposit of $10 \mu \mathrm{m}$ of $\mathrm{Cu}$ ) was placed on the surface of the blocks and poles. Copper is used as a means of conducting the return current flowing at the surface of the blocks thereby minimizing the heat deposition. Nickel is magnetic and maintains the sheet in close contact with the pole faces without any corrugation (permanent or heatload induced). The small thickness of Nickel produces a negligible reduction of the undulator field. At both upstream and downstream extremities of the magnet structures flexible transitions are installed. These provide a smooth transition between the vertical aperture of the adjacent chambers and the vertical gap in the undulator thereby minimizing any impedance discontinuity of the vacuum chamber. In addition, some $\mathrm{RF}$ masks are placed laterally all along the $1.6 \mathrm{~m}$ long undulator on both sides of the magnet structure to minimize the penetration of high order RF modes into the rest of the vacuum tank. These masks are equipped with holes to provide sufficient pumping conductance.

\subsection{Vacuum System}

The vacuum tank is equipped with two $230 \mathrm{l} / \mathrm{s}$ and one $4001 / \mathrm{sec}$ ion pumps as well as two Titanium sublimators each $1000 \mathrm{l} / \mathrm{sec}$. Before inserting the magnetic assemblies, the vacuum tank and pumps were baked at $200{ }^{\circ} \mathrm{C}$ for 20 days. A number of bake-outs were later performed with the magnet assemblies in place at a maximum temperature of $120{ }^{\circ} \mathrm{C}$ for several days. Fig. 2 shows the pressure recorded in three places inside the vacuum system, normalized to the circulating ring current $(\mathrm{mbar} / \mathrm{mA})$, as a function of the time integrated ring current (A.h). Such plots are routinely performed at the ESRF following any replacement of a vacuum chamber. As a result of extensive cleaning, baking and pumping, a rapid conditioning of the undulator chamber was possible, thereby making it immediately available to the users without Bremsstrahlung down to a magnetic gap of 8 $\mathrm{mm}$.

On both magnet jaws, some coaxial thermocouples were fitted on the flexible upstream and downstream transition sections as well as on the poles. As expected, the highest temperature of $120{ }^{\circ} \mathrm{C}$ was observed at the junction between the flexible transition and the magnet assembly. However, over the first two months of operation the thermocouples, placed on the poles at $1 / 3$ and $2 / 3$ length of the undulators, never exceeded $40^{\circ} \mathrm{C}$.

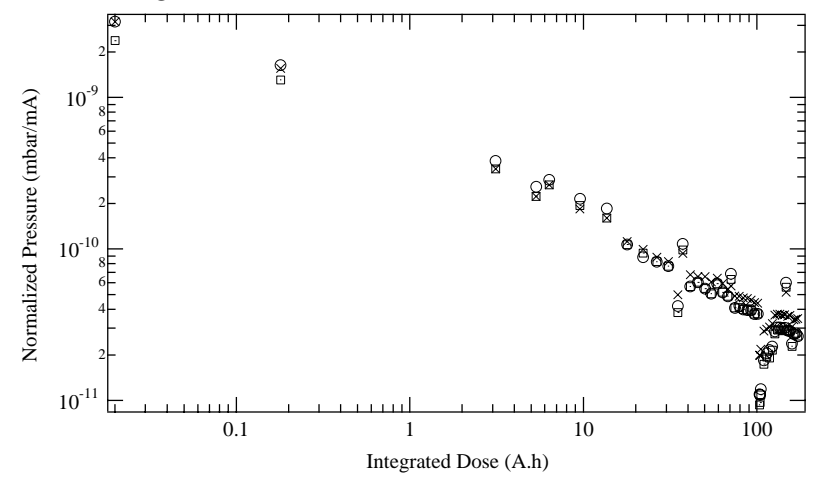

Figure 2: Vacuum conditioning curves

\subsection{Effect on the electron beam}

Due to a lack of time, it was not possible to correct and shim the magnet structure in the same way as other conventional undulators. As a consequence, a residual field integral of $150 \mathrm{Gcm}$ was observed and later corrected with coils. No lifetime reduction was detected beyond 43 hours ( 8 hours) when the magnetic gap was reduced to 6 $\mathrm{mm}(5 \mathrm{~mm})$ in the $200 \mathrm{~mA} 2 / 3$ filling mode $(80 \mathrm{~mA} 16$ bunch mode). Due to a heatload limitation in the front-end part of the beamline, the minimum gap for user operation at $200 \mathrm{~mA}$ has been left for the moment at $8 \mathrm{~mm}$, however, it is likely that it will be reduced to $6 \mathrm{~mm}$ in the near future. These lifetime observations are not surprising since the lifetime is defined by two scrapers permanently closed at $5 \mathrm{~mm}$ during the user mode of operation.

\subsection{X-Ray Spectrum}

Fig. 3 presents the undulator spectral flux computed with SRW [3] for a gap of $8 \mathrm{~mm}$ and $6 \mathrm{~mm}$. Some overlap of the harmonics is observed due to the small horizontal beta function of the ring lattice at the source $(0.5 \mathrm{~m})$ which results in a large angular spread of the electron beam. An angular spectral flux six times higher and narrower harmonics are expected if this undulator is placed on an even straight section where the horizontal beta function is large $(36 \mathrm{~m})$.

Again, due to a lack of time, an unusually large phase error was left, resulting in a reduction of the angular flux per unit surface on the peak of the harmonics. Compared to an ideal undulator, the angular flux on harmonics $1,3,5$ and 7 is expected to be $0.97,0.74,0.6$ and 0.5 (respectively). 


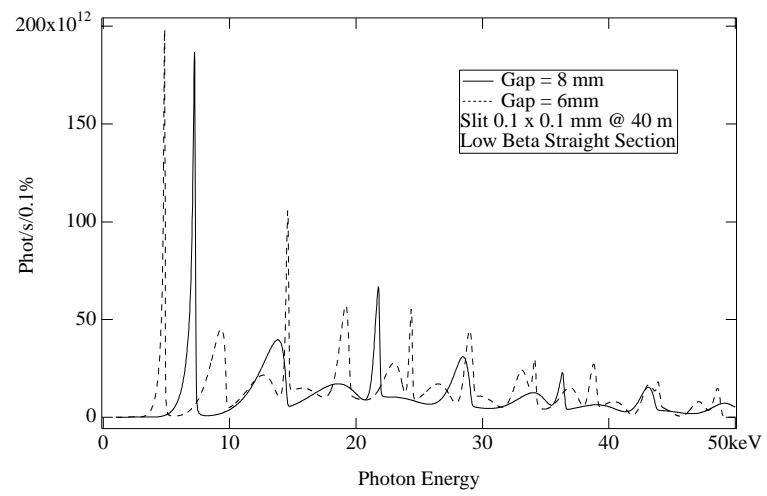

Figure 3: Spectrum through a 0.1 x $0.1 \mathrm{~mm}$ aperture located at $40 \mathrm{~m}$ from the source computed for an 8 and 6 mm gap

\section{SPECIAL IDS}

\subsection{Three Tesla Wiggler}

A 3 Tesla asymmetric wiggler, made of two periods of $378 \mathrm{~mm}$, has been built [4]. It is essentially a hybrid design with poles made of VACOFLUX 50 and magnets made of VACODYM 383HR. The magnetic design was made in 3D using the RADIA code [5]. The measured peak field reaches $3.13 \mathrm{~T}$ for a gap of $11 \mathrm{~mm}$ (see Fig. 4) and $3.57 \mathrm{~T}$ for a gap of $6 \mathrm{~mm}$. The advantage of such IDs is their low cost compared to a superconducting magnet.

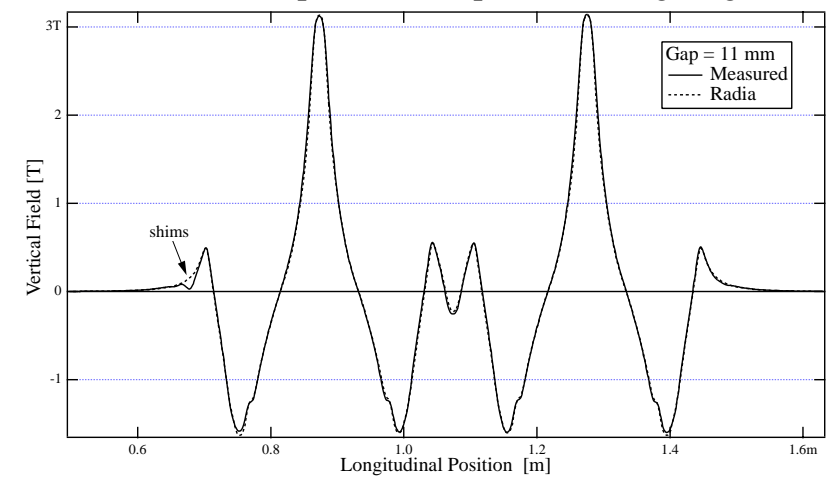

Figure 4: Field Plot of the 3 Tesla wiggler measured at a gap of $11 \mathrm{~mm}$.

\subsection{Quasi -Periodic Undulator}

A novel and simple design of a quasi-periodic undulator has been tested [6]. It is derived from a conventional pure permanent magnet undulator design by shifting a few magnet blocks vertically. Its main advantage is its simplicity and high spectral flux per unit length. A rejection ratio greater than 8 (11) has been observed on harmonics 3 and 5. With the experience gained from this prototype, a $54 \mathrm{~mm}$ period quasi-periodic undulator was designed, and is now undergoing final tests. The fundamental harmonic of this device covers the 1-6 keV energy range with a large suppression of harmonics 3 to 9.

\subsection{Fast Switching Helical Undulator}

A new fast switching linear/helical undulator has been built and measured [7]. The vertical field of this ID is produced by a coil and a laminated iron structure while the horizontal field is produced by an array of permanent magnets located between the poles. The spatial period is $80 \mathrm{~mm}$ and its length is $1600 \mathrm{~mm}$ with a peak field around 0.2 Tesla. A fast flipping of the circular polarization from left to right is induced by reversing the current from +250 A to -250 A within a time span which can be as short as $6 \mathrm{~ms}$. Initial tests have shown that a dichroism signal as low as $10^{-4}$ can be detected within a few minutes of integration time [8]. This device opens many new scientific opportunities.

\section{ACKNOWLEDGMENTS}

The authors would like to thank the Vacuum Group and the Drafting Office for their help in building the invacuum undulator and to all the members of the Insertion Device Group for their daily support. We would like also to thank the Spring8 team for a number of valuable discussions concerning all aspects of the engineering of in-vacuum undulators.

\section{REFERENCES}

[1] P. Colomp, T. Oddolaye, P. Elleaume, "Demagnetization of Permanent Magnets to $180 \mathrm{MeV}$ Electron Beam", ESRF Internal Note, ESRF/MACH-ID/93-09 and J. Chavanne, P. Elleaume, P. Van Vaerenbergh, "Ageing of Permanent Magnet Devices at the ESRF", to be published at RADECS 99

[2] P. Van Vaerenbergh, Internal ESRF Note, PVV/memo/98-44

[3] O. Chubar, P. Elleaume, "Accurate And Efficient Computation Of Synchrotron Radiation In The Near Field Region", proc. of the EPAC98 Conference 1177-1179 (1998). SRW is freely available from

" http://www.esrf.fr/machine/support/ids/Public/Codes/software.html"

[4] J Chavanne, P Van Vaerenbergh and P Elleaume, "A 3 Tesla Asymmetric Permanent Magnet Wiggler”, NIM A 421 (1999) 352-360

[5] P. Elleaume, O. Chubar, J. Chavanne, "Computing 3D Magnetic Field from Insertion Devices", Proc. of PAC97 Conference, p.35093511 (1997). Available from: "http://www.esrf.fr/machine/support/ids/ Public/Codes/software.html"

[6] J. Chavanne, P. Elleaume, P. Van Vaerenbergh, "Development Of Quasiperiodic Undulators at the ESRF", Proc. of the EPAC98 Conference 2213-2215 (1998).

[7] J. Chavanne, P. Elleaume, P. Van Vaerenbergh, "A Novel Fast Switching Linear/Helical Undulator", Proc. of the EPAC98 Conference p.317-319 (1998)

[8] A.Rogalev et al., To be submitted to SPIE. 